\title{
Prevalência e fatores associados a amputações por pé diabético
}

\author{
The prevalence and factors associated with diabetic foot amputations
}

Isabel Cristina Ramos Vieira Santos ${ }^{1}$

Christiana Maria Maia Sobreira ${ }^{1}$

Émilli Natália dos Santos Nunes ${ }^{1}$

Maria Carolina de Andrade Morais ${ }^{1}$

${ }^{1}$ Faculdade de Enfermagem Nossa Senhora das Graças, Universidade de

Pernambuco. R. Arnóbio

Marques 310, Santo Amaro. 50.100-130 Recife PE.

tutornad@yahoo.com.br

\begin{abstract}
The scope of this investigation was to establish the prevalence of diabetic foot amputations and analyze associations with factors related to the individual and to primary health care. It is a cross-sectional epidemiological study and the sample included 214 inpatients with diabetic feet in one of the four hospitals specialized in vascular surgery in the state of Pernambuco. Data were collected using semi-structured questionnaires. In the data analysis, chi-square association, Poisson regression $(p<0.05)$ and descriptive statistics procedures were applied. The prevalence of amputation ascertained was 50\%. The following factors related to the individual were associated with its occurrence: low education ( $p=0.027)$; two or more people living in the household ( $p=0.046)$; and patient income below the minimum wage ( $p=$ 0.004). The following factors related to primary health care were associated with amputation: not having feet examined $(P R=1.17)$; not receiving guidance on foot care $(P R=2.24)$ in consultations in the past year; not using the drug for DM control as prescribed $(P R=1.60)$; and inadequate glycemic control $(P R=1.83)$. Understanding these factors helps to identify aspects of preventive care that require improvement.
\end{abstract}

Key words Diabetic foot, Amputations, Primary health care
Resumo O objetivo desta investigação foi determinar a prevalência de amputações por pé diabético e analisar associações com fatores relacionados à pessoa e à atenção básica. Estudo epidemiológico de corte transversal. A amostra compreendeu 214 portadores de pé diabéticos internados em um dos quatro hospitais com especialidade cirurgia vascular de Pernambuco. Os dados foram coletados mediante questionário semiestruturado. Na análise dos dados foram aplicados os procedimentos da estatística descritiva, análise de associações de qui-quadrado e de regressão de poisson $(p<0,05)$. A prevalência de amputações encontrada foi de 50\%. Estiveram associados à sua ocorrência fatores relacionados à pessoa: baixa escolaridade ( $p=0,027)$, duas ou mais pessoas residentes no domicílio $(p=0,046)$ e renda do paciente inferior a um salário mínimo $(p=0,004)$. Dos fatores relacionados à atenção básica: não ter os pés examinados $(R P=1,17)$ e não ter recebido orientações sobre os cuidados com os pés ( $R P=$ 2,24) nas consultas realizadas no último ano, além de não usar o medicamento para controle do DM conforme prescrição $(R P=1,60)$ e controle inadequado da glicemia $(R P=1,83)$ foram associados à ocorrência de amputações. A compreensão desses fatores ajuda a identificar aspectos da assistência preventiva que precisam ser melhorados. Palavras-chave Pé diabético, Amputações, Atenção básica 


\section{Introdução}

Diabetes mellitus (DM) é uma doença conhecida por suas complicações multifacetadas, dentre as quais uma das mais comuns é o pé diabético ${ }^{1}$.

As complicações de extremidades inferiores tem se tornado um crescente e significante problema de saúde pública tanto em países desenvolvidos quanto naqueles em desenvolvimento. Essas complicações se associam com neuropatia e doença arterial periférica que predispõem ou agravam as lesões nos pés, levando subsequentemente à infecção e amputação $0^{1-3}$.

A prevalência de úlceras nos pés atinge $4 \%$ a $10 \%$ das pessoas portadoras de diabetes. Cerca de $40 \%$ a $60 \%$ das amputações não traumáticas de membros inferiores ocorrem nesses pacientes sendo que $85 \%$ destas são precedidas de úlceras nos pés ${ }^{4-5}$.

A amputação está associada com significantes custos e pode ter repercussões a longo termo, tais como o risco elevado para reulceração, perda da mobilidade e diminuição da qualidade de vida $^{5}$.

Esta síndrome guarda relação com o tempo de duração do Diabetes e consequentemente com a idade. A demora no início do tratamento adequado aumenta a ocorrência de complicações e a necessidade de amputação ${ }^{4}$.

Existem importantes diferenças nas taxas de amputação entre diversos serviços na Europa. Estudo realizado em 10 países europeus demonstrou uma variação de 2,4 a $34 \%$ e afirma que tal variação pode ser explicada em parte pela severidade da doença durante a internação, sugerindo que o referenciamento precoce a serviços especializados pode prevenir amputações ${ }^{5}$.

Ações em saúde, efetivas, no cuidado com os pés, visando à prevenção do pé diabético poderiam evitar $44 \%$ a $85 \%$ das amputações. Soma-se a isto o estímulo ao autocuidado, o atendimento interdisciplinar e a educação em saúde ${ }^{4}$.

Estudo de âmbito nacional sobre internações por Diabetes mellitus observou uma razão de chances de óbito hospitalar duas vezes maior para habitantes das regiões Nordeste e Sudeste comparados aos da região Sul e enfatiza que o manejo adequado do DM no nível de atenção básica reduziria os efeitos econômicos adversos para famílias, comunidades e sociedade em geral, provocadas por internações e, principalmente, reduziriam as sequelas e complicações ${ }^{6}$.

Os programas federais de atenção aos portadores desta patologia têm por pressuposto que o seguimento de rotina dos pacientes deve ser realizado na porta de entrada dos serviços, que têm como eixo estruturante a Estratégia de Saúde da Família (ESF) 7 .

Uma melhor compreensão dos fatores que estão relacionados à ocorrência de amputações em indivíduos com pé diabético poderá ajudar a identificar aspectos do cuidado desses pacientes que necessitam ser melhorados ${ }^{5}$.

Fatores associados a amputações são pouco conhecidos e podem incluir características relacionadas ao paciente ou à úlcera, mas também, a fatores externos tais como práticas dos profissionais envolvidos no cuidado e/ou aspectos da organização dos serviços de saúde ${ }^{5}$.

Na perspectiva da problemática do acesso aos serviços tendo como foco a ESF, as dimensões organizacionais e ou funcionais parecem ser mais relevantes devido à própria forma de organização dos serviços em que os esforços pela mudança do modelo de atenção, tendendo do hospitalocêntrico e medicalizante para a atenção primária centrada em equipes multidisciplinares é uma realidade ${ }^{7}$.

A magnitude do Diabetes e suas complicações, especificamente o pé diabético, no cenário nacional e a responsabilidade do manejo dessa condição pela atenção básica motivaram este trabalho, que teve por objetivos: estimar a prevalência de amputações por pé diabético e verificar associação com fatores relacionados à atenção básica.

\section{Métodos}

A cidade do Recife é o município mais populoso do Estado de Pernambuco (1.536.934 habitantes) e sede da região metropolitana que se estende por mais treze municípios, com uma população total de 3.688.428 habitantes.

O serviço de saúde do município compreende 4 hospitais pertencentes ao Sistema Único de Saúde com especialidade em cirurgias vasculares e com internação ${ }^{8}$.

Este estudo de abordagem epidemiológica descritiva foi desenvolvido em um hospital público escolhido devido à sua localização estratégica e ao número de leitos (40) destinados à cirurgia vascular. Atende à população proveniente da região metropolitana e de municípios do interior do estado de Pernambuco, com uma média anual de internamentos na clínica vascular da ordem de 561,7 pacientes.

Foram incluídos todos os indivíduos portadores de pé diabéticos internados na clínica vas- 
cular para tratamento clínico ou cirúrgico no período de 01/07/2008 a 01/07/2010.

$\mathrm{O}$ procedimento inicial consistiu na busca diária de internamentos de portadores de pé diabético, utilizando-se para isto o censo da referida clínica e os respectivos prontuários para seleção dos pacientes de interesse ao estudo, após o que se realizou entrevista com os mesmos, utilizando-se um formulário.

O formulário foi construído a partir do Consenso Internacional sobre pé diabético ${ }^{9}$ e do Manual de hipertensão arterial e diabetes mellitus ${ }^{10}$, instrumento normativo do Plano de Reorganização da Atenção à Hipertensão arterial e ao Diabetes mellitus, utilizado pelo Ministério da Saúde para reorganização da atenção básica a essas duas doenças.

As variáveis investigadas corresponderam além da ocorrência de amputações, as de caracterização da pessoa: faixa etária, sexo, procedência, anos de estudo, $\mathrm{n}^{\circ}$ de pessoas no domicílio, renda do paciente, renda familiar, presença de vasculopatia e presença de neuropatia. Também fatores relacionados à atenção básica: consultas no último ano, glicemia, exame dos pés nas consultas do último ano, orientação sobre os cuidados com os pés, uso de medicamento para DM e disponibilidade de medicamentos.

Todos os testes foram aplicados com 95\% de confiança. Para verificar a existência de associação entre as variáveis categóricas foi utilizado o teste Qui-quadrado.

As variáveis numéricas estão representadas pelas medidas de tendência central e de dispersão. O teste de normalidade de KolmogorovSmirnov foi utilizado e para comparação com dois grupos: Teste t Student (Distribuição Normal) e Mann-Whitney (Não Normal).

Adotou-se o modelo de Regressão de Poisson para identificar aspectos associados significativamente à variável de desfecho, ajustando-se concomitantemente o efeito de uma variável pelo das demais de interesse incluídas na análise. Para a pré-seleção das covariáveis, observaram-se aquelas que apresentavam valor $\mathrm{p}$ menor ou igual a 0,20 na análise bivariada e em seguida incluídas no modelo final. Todas as conclusões foram tomadas para $\mathrm{p}<0,05$.

O software SPSS na versão 13.0 foi utilizado para digitação dos dados, com dupla entrada e para as análises de variáveis categóricas e contínuas. Para a regressão foi utilizado o Software STATA/SE 9.0

O projeto desta pesquisa foi aprovado pelo Comitê de Ética em Pesquisa do referido hospital.

\section{Resultados}

Entre 2008 e 2010 foram internados 214 portadores de pé diabéticos no hospital estudado. Destes $107(50 \%)$ foram submetidos à amputação de algum segmento dos membros inferiores.

A idade média foi de 65 anos (mediana $=65$ anos; DP $=11,6)$. O teste de associação entre as variáveis categóricas (Tabela 1) mostrou significância estatística $(\mathrm{p}<0,05)$ para as variáveis: anos de estudo, onde se observa que $64,5 \%$ dos que realizaram amputação tinham entre 0 a 4 anos de estudo; dentre os pacientes que realizaram amputação o número de 2 ou mais pessoas residentes no domicílio esteve associada ao referido evento, correspondendo a 91,6\% dos pacientes submetidos a esse procedimento; a renda do paciente de até um salário mínimo correspondeu a 92,5\% dos pacientes amputados e a vasculopatia e neuropatia estiveram presentes respectivamente em $63,6 \%$ e $59,8 \%$ destes.

Segundo se apresenta na Tabela 2, foram verificadas associações $(\mathrm{p}<0,05)$ entre algumas variáveis da Atenção básica tais como: número de consultas no ultimo ano, realização de glicemia no último ano, conhece o valor normal da glicemia, recebeu informação sobre resultado da glicemia, exame dos pés nas consultas do último ano, recebeu orientação sobre os cuidados com os pés nas consultas realizadas, faz uso de medicamento para DM e glicemia de admissão em relação ao desfecho amputação. Este desfecho foi mais prevalente entre aqueles que: receberam o diagnóstico de diabetes por outros problemas que não o dos pés $(59,8 \%)$; realizaram até três consultas no último ano (53,5\%); não realizaram glicemia no último ano $(54,8 \%)$; não conhecem o valor normal da glicemia (55,2\%); não receberam informação sobre resultado da glicemia $(61,4 \%)$; não tiveram os pés examinados nas consultas do último ano (61,3\%); não receberam orientação sobre os cuidados com os pés nas consultas realizadas (55,4\%); não fazem uso de medicamento para DM conforme orientação medica $(67,2 \%)$ e que apresentaram valores de glicemia superiores a $126 \mathrm{mg} / \mathrm{dl}$ à admissão (56,9\%).

A comparação entre os pacientes que realizaram amputação e os que não foram submetidos a este procedimento, segundo variáveis relacionadas à pessoa e à atenção básica (Tabela 3) mostrou diferenças estatisticamente significativas para a média de consultas realizadas no último ano menor que três $(1,7)$ e média de glicemia à admissão de 224,0. 
Tabela 1. Fatores relacionados à pessoa associados à amputação por pé diabético. Recife, Pernambuco, Brasil, 2010.

\begin{tabular}{|c|c|c|c|}
\hline \multirow[b]{2}{*}{ Variáveis } & \multicolumn{2}{|c|}{ Amputação (\%) } & \multirow[b]{2}{*}{ p-valor } \\
\hline & $\operatorname{Sim} \mathbf{n}(\%)$ & Não n (\%) & \\
\hline \multicolumn{4}{|l|}{ Faixa Etária } \\
\hline$<60$ anos & $47(43,9 \%)$ & $39(36,4 \%)$ & 0,329 \\
\hline 60 ou mais anos & $60(56,1 \%)$ & $68(63,6 \%)$ & \\
\hline \multicolumn{4}{|l|}{ Sexo } \\
\hline Masculino & $63(58,9 \%)$ & $54(50,5 \%)$ & 0,272 \\
\hline Feminino & $44(41,1 \%)$ & $53(49,5 \%)$ & \\
\hline \multicolumn{4}{|l|}{ Procedência } \\
\hline RMR & $80(74,8 \%)$ & $74(69,2 \%)$ & 0,447 \\
\hline Interior/Outros Estados & $27(25,2 \%)$ & $33(30,8 \%)$ & \\
\hline \multicolumn{4}{|l|}{ Anos de Estudo } \\
\hline $0-4$ anos & $69(64,5 \%)$ & $52(48,6 \%)$ & 0,027 \\
\hline$>4$ anos & $38(35,5 \%)$ & $55(51,4 \%)$ & \\
\hline \multicolumn{4}{|l|}{ Pessoas no domicílio } \\
\hline 1 pessoa & $9(8,4 \%)$ & $20(18,7 \%)$ & 0,046 \\
\hline 2 ou mais pessoas & $98(91,6 \%)$ & $87(81,3 \%)$ & \\
\hline \multicolumn{4}{|l|}{ Renda } \\
\hline Até $1 \mathrm{SM}$ & $99(92,5 \%)$ & $83(77,6 \%)$ & 0,004 \\
\hline Mais de $1 \mathrm{SM}$ & $8(7,5 \%)$ & $24(22,4 \%)$ & \\
\hline \multicolumn{4}{|l|}{ Renda Familiar } \\
\hline Até $1 \mathrm{SM}$ & $72(67,9 \%)$ & $66(63,5 \%)$ & 0,592 \\
\hline Mais de $1 \mathrm{SM}$ & $34(32,1 \%)$ & $38(36,5 \%)$ & \\
\hline \multicolumn{4}{|l|}{ Vasculopatia } \\
\hline $\operatorname{Sim}$ & $68(63,6 \%)$ & $48(44,9 \%)$ & 0,009 \\
\hline Não & $39(36,4 \%)$ & $59(55,1 \%)$ & \\
\hline \multicolumn{4}{|l|}{ Neuropatia } \\
\hline Sim & $64(59,8 \%)$ & $40(37,4 \%)$ & 0,002 \\
\hline Não & $43(40,2 \%)$ & $67(62,6 \%)$ & \\
\hline
\end{tabular}

* Teste de Qui-Quadrado.

Na Tabela 4, é apresentada a associação entre amputação por pé diabético com as variáveis relacionadas à Atenção Básica. Os resultados indicaram que os pacientes que: não tiveram os pés examinados nas consultas do último ano (RP: 1,17; IC95\%: 1,07; 1,29), não receberam orientação sobre os cuidados com os pés nas consultas realizadas (RP: 2,24; IC95\%: 1,51; 3,31), não faziam uso de medicamento para DM conforme prescrição médica (RP: 1,60; IC95\%: 1,25;2,04) e com valores de glicemia superiores a $126 \mathrm{mg} / \mathrm{dl}$ à admissão (RP: 1,83; IC95\%: 1,25; 2,69) apresentaram maior probabilidade de sofrerem uma amputação.

\section{Discussão}

No presente estudo, observou-se que 50\% dos portadores de pé diabéticos internados foram submetidos a alguma amputação de membros inferiores. Sobre isto não existem parâmetros recentes para comparação no país. A prevalência aqui encontrada corresponde àquela informada no Quênia, que varia entre 25\% e 56\% ${ }^{3}$.

Este é, segundo nosso conhecimento, um dos poucos estudos no Brasil que avalia a prevalência de amputações por pé diabético e, embora tenha como principal limitação a ser considerado nesta discussão o fato de ser uma pesquisa de base hospitalar, os cuidados tomados para a coleta dos dados feita durante os dois anos de seguimento das internações, evitando a possibilidade de erro que uma amostragem realizada em certas épocas do ano poderia trazer, além de cor- 
Tabela 2. Prevalência de amputações por pé diabético de acordo com as características da Atenção básica. Recife, Pernambuco, Brasil, 2010.

\begin{tabular}{|c|c|c|c|c|}
\hline Variáveis & Amputação (\%) & n (214) & IC & $\mathrm{p}$ \\
\hline \multicolumn{5}{|c|}{ Momento em que teve diagnóstico de DM } \\
\hline Consulta & 45,5 & 123 & \multirow{2}{*}{$0,96-1,63$} & \multirow{2}{*}{0,105} \\
\hline Internação & 56,8 & 88 & & \\
\hline \multicolumn{5}{|l|}{ Consultas no último ano } \\
\hline Sim & 44,7 & 123 & \multirow{2}{*}{$0,98-1,67$} & \multirow{2}{*}{0,072} \\
\hline Não & 57,1 & 91 & & \\
\hline \multicolumn{3}{|l|}{ Número de consultas } & \multirow{3}{*}{$1,09-3,00$} & \multirow{3}{*}{0,011} \\
\hline $1-3$ & 53,5 & 71 & & \\
\hline$>3$ & 29,5 & 44 & & \\
\hline \multicolumn{5}{|l|}{ Realização de glicemia no último ano } \\
\hline Sim & 40,3 & 72 & \multirow{2}{*}{$0,53-1,01$} & \multirow{2}{*}{0,046} \\
\hline Não & 54,8 & 135 & & \\
\hline \multicolumn{5}{|l|}{ Conhece o valor normal da glicemia } \\
\hline Sim & 41,3 & 80 & \multirow{2}{*}{$0,99-1,81$} & \multirow{2}{*}{0,047} \\
\hline Não & 55,2 & 134 & & \\
\hline \multicolumn{5}{|c|}{ Recebeu informação sobre resultado da glicemia } \\
\hline Sim & 42,1 & 126 & \multirow{2}{*}{$1,12-1,90$} & \multirow{2}{*}{0,005} \\
\hline Não & 61,4 & 88 & & \\
\hline \multicolumn{5}{|c|}{ Exame dos pés nas consultas do último ano } \\
\hline Sim & 44,0 & 125 & \multirow{2}{*}{$0,55-0,94$} & \multirow{2}{*}{0,016} \\
\hline Não & 61,3 & 80 & & \\
\hline \multicolumn{5}{|c|}{$\begin{array}{l}\text { Recebeu orientação sobre os cuidados com os pés nas } \\
\text { consultas realizadas }\end{array}$} \\
\hline Sim & 35,1 & 57 & \multirow{2}{*}{$1,08-2,31$} & \multirow{2}{*}{0,009} \\
\hline Não & 55,4 & 148 & & \\
\hline \multicolumn{5}{|c|}{ Recebeu orientação nutricional nas consultas realizadas } \\
\hline Sim & 45,9 & 135 & \multirow{2}{*}{$0,97-1,67$} & \multirow{2}{*}{0,085} \\
\hline Não & 58,6 & 70 & & \\
\hline \multirow{2}{*}{\multicolumn{5}{|c|}{$\begin{array}{l}\text { Recebeu orientação sobre atividade física nas consultas } \\
\text { realizadas }\end{array}$}} \\
\hline & & & & \\
\hline Não & $\begin{array}{l}45,0 \\
55,0\end{array}$ & $\begin{array}{r}90 \\
109\end{array}$ & $0,95-1,67$ & 0,106 \\
\hline Faz uso de medicamento para DM & & & & \\
\hline Sim & 43,1 & 153 & & 0001 \\
\hline Não & 67,2 & 61 & $1,21-2,01$ & 0,001 \\
\hline Glicemia de admissão & & & & \\
\hline$<126 \mathrm{mg} / \mathrm{dl}$ & 32,1 & 56 & & \\
\hline $126 \mathrm{mg} / \mathrm{dl} \mathrm{a}+$ & 56,9 & 153 & $1,18-2,65$ & 0,001 \\
\hline
\end{tabular}

responder a cerca de $30 \%$ da clientela assistida nos hospitais do SUS no Estado de Pernambuco (1/4 hospitais), lhe confere credibilidade.

A prevalência de amputações encontrada neste estudo mostrou-se associada às seguintes variáveis relacionadas à pessoa: baixa escolaridade, número de pessoas residentes no domicílio correspondente a 2 ou mais e renda inferior a um salário mínimo. Resultados coerentes com o que afirma estudo realizado em Maceió (AL) de que estas amputações são mais frequentes na população diabética de baixo nível socioeconômico ${ }^{11}$.
Também em relação à baixa renda, se assemelha ao resultado de estudo realizado em Recife $(\mathrm{PE})^{12}$. O número de habitantes no domicilio tem implicação direta sobre o controle da doença, no que se refere à alimentação e aos cuidados básicos de higiene e de calçados, uma vez que uma renda de até um salário mínimo ( $\mathrm{R}$ \$ 510,00 em 2010), dividida por 2 ou mais habitantes, independentemente da idade e da situação de saúde, equivale ao conceito, dado pelo Banco Mundial, de pobreza moderada, ou seja, viver com entre 1 e 2 dólares por dia. 
Tabela 3. Fatores associados com amputações por pé diabético. Recife, Pernambuco, Brasil, 2010.

\begin{tabular}{lccc}
\hline \multirow{2}{*}{\multicolumn{1}{c}{ Variáveis }} & \multicolumn{2}{c}{ Amputação (\%) } & \\
\cline { 2 - 3 } & Sim & Não & p-valor \\
& Média \pm DP & Média \pm DP & \\
\hline Idade & $64,2 \pm 11,703$ & $65,8 \pm 11,517$ & $0,312^{* *}$ \\
Anos de estudo & $4,1 \pm 3,880$ & $3,3 \pm 3,582$ & $0,124^{* *}$ \\
No de pessoas que residem no domicílio & $4,0 \pm 2,363$ & $3,6 \pm 2,372$ & $0,065^{* *}$ \\
Renda do paciente & $471,0 \pm 514,597$ & $426,0 \pm 169,171$ & $0,996^{* *}$ \\
Renda familiar & $693,8 \pm 523,904$ & $652,7 \pm 406,960$ & $0,980^{* *}$ \\
Número de internamentos por problemas com os pés & $1,8 \pm 0,752$ & $1,8 \pm 0,676$ & $0,742^{* *}$ \\
Número de consultas no último ano & $1,7 \pm 2,304$ & $2,8 \pm 3,169$ & $\mathbf{0 , 0 2 8}$ \\
Resultado da glicemia a admissão & $224,0 \pm 90,918$ & $190,8 \pm 87,965$ & $\mathbf{0 , 0 0 1}$ \\
\hline
\end{tabular}

${ }^{*}$ Teste t-Student. ${ }^{* *}$ Teste de Mann-Whitney.

Tabela 4. Análise de regressão de Poisson entre amputação por pé diabético e variáveis da Atenção básica. Recife, Pernambuco, Brasil, 2010.

\begin{tabular}{lccc}
\hline \multicolumn{1}{c}{ Variáveis } & RP & IC [95\%] & p-valor \\
\hline Exame dos pés nas consultas do último ano & & & \\
$\quad$ Sim & 1,00 & - & $<0,001$ \\
$\quad$ Não & 1,17 & {$[1,07 ; 1,29]$} & \\
Recebeu orientação sobre os cuidados com os pés nas consultas realizadas & & & \\
$\quad$ Sim & 1,00 & - & $<0,001$ \\
$\quad$ Não & 2,24 & {$[1,51 ; 3,31]$} & \\
Faz uso de medicamento conforme prescrição & & & \\
$\quad$ Sim & 1,00 & - & $<0,001$ \\
$\quad$ Não & 1,60 & {$[1,25 ; 2,04]$} & \\
Glicemia de admissão & & & \\
$\quad<126 \mathrm{mg} / \mathrm{dl}$ & 1,00 & - & 0,002 \\
$\quad>126 \mathrm{mg} / \mathrm{dl}$ & 1,83 & {$[1,25 ; 2,69]$} &
\end{tabular}

RP: razão de Prevalência; IC: intervalo de confiança.

Em adição a isso, a baixa escolaridade do diabético impõe aos profissionais da atenção básica um desafio que requer o planejamento de estratégias diferenciadas e intensivas quando das orientações para o autocuidado, de modo a alcançar efetivamente a população alvo.

Neste estudo, a vasculopatia e a neuropatia estiveram associadas à ocorrência de amputações, com prevalências de 63,6\% e 59,8\% respectivamente.

Estudo de coorte ${ }^{5}$, com 1.232 pacientes, realizado na Europa demonstrou que a doença vascular é fator predisponente para amputações em pacientes diabéticos (odds ratio: 1.84, intervalo de confiança: 1.30-2.60).

Outro estudo realizado em Madri ${ }^{13}$ encontrou que, entre diabéticos, $100 \%$ de amputações maiores, ou seja, aquelas realizadas acima do nível do pé estavam associadas com a presença de doença vascular periférica, $78 \%$ com neuropatia e $24 \%$ com infecção. Por outro lado, nos casos de amputações menores (correspondente à região abaixo do tornozelo), 62\% estavam associados com vasculopatia, $92 \%$ com neuropatia e $84 \%$ com infecção.

Estes resultados podem sugerir que o referenciamento precoce desses pacientes para o atendimento de maior complexidade do sistema de saúde pode prevenir amputações.

No que diz respeito às variáveis relacionadas à Atenção básica, os resultados aqui encontrados mostraram associação entre a ocorrência de amputações e o número de 1 a 3 consultas realizadas no último ano, tanto pela análise qualitativa quanto quantitativa.

$\mathrm{O}$ instrumento normativo do Ministério da Saúde ${ }^{10}$ para atenção à diabetes mellitus prescre- 
ve que o acompanhamento desses pacientes deve ser feito em 3 ou 4 consultas ao ano.

Pouco mais da metade dos pacientes submetidos à amputação $(54,8 \%)$ referiram não ter realizado exame de glicemia no último ano $(\mathrm{p}<$ 0,05).

Estudo sobre o acesso a serviços de saúde na Baixada Santista ${ }^{7}$, mostrou que em relação ao exame de glicemia, a maioria dos pacientes havia realizado o referido exame. Entretanto, quando da analise dos últimos 6 meses este indicador diminuiu para apenas $49,6 \%$. Os autores chamam a atenção de que a Programação Pactuada e Integrada (PPI) recomenda quatro exames de glicemia paciente/ano e que, portanto, há uma necessidade iminente de adequação dos serviços de atenção básica no cumprimento desta atividade.

Soma-se a isso, o fato de que $55,2 \%$ dos pacientes submetidos à amputação por pé diabético neste estudo referiram não saber o valor normal da glicemia e que $61,4 \%$ deles referiram não serem informados sobre os resultados do exame, ambos estatisticamente significativos. Isto pode repercutir na adesão ao tratamento, uma vez que tal conhecimento implicaria em ações de autocuidado, podendo inclusive influenciar negativamente no controle da glicemia, fator reconhecido como de risco para a amputação ${ }^{14}$.

Observou-se que $61,3 \%$ e $55,4 \%$ dos pacientes submetidos à amputação referiram não ter os pés examinados e não receber orientação sobre o cuidado com estes, respectivamente, nas consultas realizadas no último ano. Estas variáveis mostraram-se associadas à ocorrência de amputações e se mantiveram presentes no modelo final de analise multivariada, apresentando uma razão de prevalência de 1,17 e 2,24 respectivamente.

As medidas preventivas precoces e a cicatrização de úlceras diabéticas têm sido reportadas por vários estudos como fundamentais para prevenção de amputações ${ }^{1,5,12}$.

A prevenção das úlceras diabéticas pode ser alcançada na atenção básica através de breve história clínica e triagem para perda da sensação protetora e exame dos pulsos distais. Estas medidas simples e custo-efetivas ajudam a estratificar os pacientes baseados no risco e a determinar o tipo de intervenção necessária e oportuna. Além disso, atividades educativas nos serviços de saúde podem ajudar na adesão dos pacientes ao tratamento.

Estudo recente sobre o acesso de pessoas portadoras de hipertensão arterial e ou diabetes aos serviços de saúde relata que as atividades educa- tivas são praticamente inexistentes entre os pacientes cadastrados na Estratégia Saúde da Família estudada. Este tipo de cuidado é recomendado pelas sociedades brasileiras de cardiologia e de diabetes como sendo essencial para que o tratamento dessas doenças seja mais efetivo ${ }^{7}$.

O não uso de medicamentos para DM conforme prescrição e os valores de glicemia acima de $126 \mathrm{mg} / \mathrm{dl}$ apresentaram-se como fatores significativos na determinação de amputações no presente estudo, com uma razão de prevalência de 1,6 e 1,8 respectivamente.

O deficiente controle da glicemia tem sido vastamente reportado como fator de risco para amputações $^{1,14,15}$. No entanto, no que diz respeito ao uso inadequado de medicamentos para o controle da DM, não se encontrou parâmetros de comparação. Tal achado merece maiores investigações sobre a razão para este problema, que hipoteticamente poderia estar relacionado à falta de compreensão da prescrição ou a problemas sociopolíticos e culturais ${ }^{16}$.

\section{Conclusão}

A prevalência de amputações por pé diabético em um dos quatro hospitais com especialidade em cirurgia vascular e com internação pertencentes ao Sistema Único de Saúde de Pernambuco foi alta. Esta prevalência está associada a fatores socioeconômicos e da atenção básica. A compreensão desses fatores pode ajudar a identificar aspectos da assistência preventiva que precisam ser melhorados.

\section{Colaboradores}

ICR Vieira Santos participou na elaboração do projeto, análise dos resultados e revisão do artigo final. CMM Sobreira colaborou na elaboração do projeto, coordenação da pesquisa de campo, revisão da literatura. ENS Nunes e MCA Morais participaram da interpretação e análise dos dados e revisão do artigo. 


\section{Referências}

1. Wu SC, Driver VR, Wrobel JS, Armstrong DG. Foot ulcers in the diabetic patient, prevention and treatment. Vasc Health Risk Manag 2007; 3(1):65-76.

2. Santos VP, Silveira DR, Caffaro RA. Risk factors for primary major amputation in diabetic patients. Sao Paulo Med J 2006; 124(2):66-70.

3. Ogeng'o JA, Obimbo MM, King'ori J. Pattern of limb amputation in a Kenyan rural hospital. Int Orthop 2009; 33(5):1449-1453.

4. Tavares DMS, Dias FA, Araújo LR, Pereira GA. Perfil de clientes submetidos a amputações relacionadas ao diabetes mellitus. Rev Bras Enferm 2009; 62(6):825-830.

5. Van Battum P, Schaper N, Prompers L, Apelqvist J, Jude E, Piaggesi A, Bakker K, Edmonds M, Holstein P, Jirkovska A, Mauricio D, Ragnarson Tennvall G, Reike H, Spraul M, Uccioli L, Urbancic V, van Acker K, van Baal J, Ferreira I, Huijberts M. Differences in minor amputation rate in diabetic foot disease throughout Europe are in part explained by differences in disease severity at presentation. Diabet Med 2011; 28(2):199-205.

6. Rosa RS, Schmidt MI, Duncan BB, Souza MFM, Lima AK, Moura L. Internações por Diabetes Mellitus como diagnóstico principal na Rede Pública do Brasil, 1999-2001. Rev bras epidemiol 2007; 10(4):465-478.

7. Bersusa AAS, Pascalicchio ÁE, Pessoto UC, Escuder MML. Acesso a serviços de saúde na Baixada Santista de pessoas portadoras de hipertensão arterial e ou diabetes. Rev bras epidemiol 2010; 13(3):513-522.

8. Instituto Brasileiro de Geografia e Estatística (IBGE). Pesquisa Nacional por Amostra de Domicílios Acesso e Utilização dos Serviços, Condições de Saúde e Fatores de Risco e Proteção à Saúde 2008. [site na Internet]. 2008 [acessado 2011 out 6]. Disponível em: http://www.ibge.gov.br/estadosat/ temas.php?sigla $=$ pe\&tema $=$ pnad_saude_2008

9. International Working Group on the Diabetic Foot (IWGDF). International Consensus on Diabetic Foot \& Practical and Specific Guidelines on the Management and Prevention of the Diabetic Foot. Bruxelas: IWGDF; 2011.
10. Brasil. Ministério da Saúde (MS). Secretaria de Políticas de Saúde. Departamento de Ações Programáticas Estratégicas. Plano de reorganização da atenção à hipertensão arterial e diabetes mellitus: manual de hipertensão arterial e diabetes Mellitus. Brasília: MS; 2002.

11. Assumpção EC, Pitta GB, Macedo ACL, Mendonça GB, Albuquerque LCA, Lyra LCB, Timbó RM, Buarque TLL. Comparação dos fatores de risco para amputações maiores e menores em pacientes diabéticos de um Programa de Saúde da Família. J Vasc Bras 2009; 8(2):133-138.

12. Vieira-Santos ICR, Nunes ENS, Melo CA, Farias DG. Amputações por pé diabético e fatores sociais: implicações para cuidados preventivos de enfermagem. Rev Rene 2011; 12(4):684-691.

13. Calle-Pascual AL, Garcia-Torre N, Moraga I, Diaz JA, Duran A, Moñux G, Serrano FJ, Martín-Alvarez PJ, Charro A, Marañes JP. Epidemiology of non trau-matic lower extremity amputation in área 7. Madrid. Between 1989 and 1999: a population- based study. Diabetes Care 2001; 24(9):1686-1689.

14. Lowe J, Tariman JD. Lower Extremity Amputations: Black Men With Diabetes Overburdened. Adv Nurse Pract 2008; 16(11):28-37.

15. Pino AE, Taghva S, Chapman C, Bowker JH. Lowerlimb Amputations in Patients With Diabetes Mellitus. Orthopedics 2011; 34(12):885-892.

16. Paula PAB, Stephan-Souza AI, Vieira RCPA, Alves TNP. O uso do medicamento na percepção do usuário do Programa Hiperdia. Cien Saude Colet 2011; 16(5):2623-2633.

Artigo apresentado em 08/03/2012

Aprovado em 27/05/2012

Versão final apresentada em 11/06/2012 